BULLETIN OF THE

AMERICAN MATHEMATICAL SOCIETY

Volume 78, Number 5, September 1972

\title{
LIPSCHITZ FUNCTION SPACES FOR ARBITRARY METRICS
}

\author{
BY JERRY JOHNSON ${ }^{1}$ \\ Communicated by Robert G. Bartle, April 13, 1972
}

The bounded (real or complex valued) functions on a set $S$ are denoted by $l_{\infty}(S)$ while $c_{0}$ and $l_{\infty}$ denote the usual sequence spaces. For background, notation and definitions concerning Lipschitz spaces, see [3].

The purpose of this note is to announce the following:

THEOREM. Let $(S, d)$ be an infinite metric space (i.e., $S$ has infinitely many points) and suppose that $\inf _{s \neq t} d(s, t)=0$. Then $\operatorname{Lip}(S, d)$ contains a subspace isomorphic with $l_{\infty}$ and $\operatorname{lip}\left(S, d^{\alpha}\right), 0<\alpha_{0}<1$, contains a complemented subspace isomorphic with $c_{0}$ (i.e., it is the range of a continuous projection on $\left.\operatorname{lip}\left(S, d^{\alpha}\right)\right)$.

Under the hypotheses of the theorem, we obtain two corollaries that were previously unknown in general.

Corollary 1. $\operatorname{lip}\left(S, d^{\alpha}\right)$ is not complemented in $\operatorname{Lip}\left(S, d^{\alpha}\right)$.

COROllary 2. $\operatorname{lip}\left(S, d^{\alpha}\right)$ is not isomorphic to a dual space.

This also provides a proof of Theorem 2.6 in [3].

Remarks. 1. Since $l_{\infty}$ is a $P_{1}$-space (see [2, p. 94]) the subspace of $\operatorname{Lip}(S, d)$ isomorphic to $l_{\infty}$ is complemented.

2. In case $\inf _{s \neq t} d(s, t)>0$, it is shown in [3, Lemma 2.5] that $\operatorname{Lip}(S, d)$ $=\operatorname{lip}(S, d)=l_{\infty}(S)$.

3. If $\operatorname{lip}\left(S, d^{\infty}\right)$ is separable, the subspace isomorphic with $c_{0}$ is automatically complemented (see [2, p. 96]). It has been shown by K. deLeeuw and T. M. Jenkins that the dual of lip $\left(S, d^{\alpha}\right)$, and hence the space itself, is separable when $S$ is compact (see [3, Theorem 4.5]). It is unknown for exactly which metric spaces $\operatorname{lip}\left(S, d^{\infty}\right)$ [resp. its dual] is separable. Let us only mention that if $S$ is the unit ball of the sequence space $l_{1}$ and $d$ is the norm restricted to $S$, then $\operatorname{lip}\left(S, d^{\alpha}\right), 0<\alpha<1$, is not separable. Also, see the example at the end of this paper.

It was shown in [1] that if $S$ is an infinite compact subset of Euclidean space and $0<\alpha<1$, then $\operatorname{lip}\left(S, d^{\alpha}\right)$ and $\operatorname{Lip}\left(S, d^{\alpha}\right)$ are isomorphic to $c_{0}$

AMS 1970 subject classifications. Primary 46E99.

1 ACKNOWLeDgements. The author is grateful to Robert P. Kaufman for suggesting the generality of the theorem and for pointing out an earlier error. He also thanks John Jobe and Forrest Whitfield for helpful conversations. 
and $l_{\infty}$ respectively. It is still an open question whether this is true for more general $S$.

We will next sketch the proof of the theorem in the case where $S$ has no nonconstant Cauchy sequences. The other case will appear elsewhere along with other results. Although the proofs are similar, the difference is substantial enough to require a careful consideration of two separate cases.

If $\left\{s_{n}\right\}$ is a sequence in $S$, there is a $\delta>0$ and a subsequence $\left\{s_{n_{k}}\right\}$ such that $d\left(s_{n_{k}}, s_{n_{l}}\right) \geqq \delta$ if $k \neq l$. This follows from the fact that $\left\{s_{n}\right\}$ is not precompact.

Let $\left\{s_{n}\right\}$ and $\left\{t_{n}\right\}$ be sequences in $S$ such that $s_{n} \neq t_{n}$ for each $n$ and $d\left(s_{n}, t_{n}\right) \rightarrow 0$. By the last remark, we may assume $d\left(s_{n}, s_{m}\right) \geqq \delta>0$ for $n \neq m$. For each $j$, let $r_{j}=\frac{1}{2} d\left(s_{j}, t_{j}\right), B_{j}=\left\{s \mid d\left(s, s_{j}\right) \leqq r_{j}\right\}$, and $d(A, B)$ $=\inf \{d(s, t) \mid s \in A, t \in B\}$.

It can now be shown that

$$
\begin{gathered}
d\left(B_{i}, B_{j}\right) \geqq \frac{1}{2} \delta \quad \text { if } i \neq j ; \\
t_{n} \notin \bigcup_{i} B_{i} \quad \text { for each } n ; \\
\frac{d\left(s, \widetilde{B}_{i}\right)+d\left(t, \widetilde{B}_{j}\right)}{d\left(B_{i}, B_{j}\right)} \leqq 3 \delta \quad \text { for } s, t \in S \text { and } i \neq j .
\end{gathered}
$$

(Here $\widetilde{B}$ denotes the complement of $B$ in $S$.)

By taking $\delta$ small enough, we may suppose $d\left(s, \widetilde{B}_{j}\right) \leqq 1$ for each $j$. We first treat the case $\alpha<1 . \alpha=1$ is special since $d^{\beta}$ is not in general subadditive for $\beta>1$. Choose $\alpha<B_{j} \leqq 1$ with $B_{j} \rightarrow \alpha$ so that $r_{j}^{\beta_{j}-\alpha}$ and $d^{\beta_{j}-\alpha}\left(s_{j}, \widetilde{B}_{j}\right)$ are $\geqq \frac{1}{2}$ for each $j$. Define $f_{j}(s)=d^{\beta_{j}}\left(s, \widetilde{B}_{j}\right)$ and for $a \in l_{\infty}$, let

$$
f_{a}=\sum_{j} a_{j} f_{j}
$$

It is not very difficult to see, using (1), (2) and (3), that $\left\|f_{a}\right\|_{\infty} \leqq\|a\|$ and $\left\|f_{a}\right\|_{d^{\alpha}} \leqq\|a\| \max \left(1,2^{1+\alpha} \delta^{-\alpha}\right)$.

Now,

$$
\begin{aligned}
\left\|f_{a}\right\|_{d \alpha} & \geqq \frac{\left|f_{a}\left(s_{j}\right)-f_{a}\left(t_{j}\right)\right|}{d^{\alpha}\left(s_{j}, t_{j}\right)}=\frac{\left|a_{j}\right| d^{\beta_{j}}\left(s_{j}, \widetilde{B}_{j}\right)}{d^{\alpha}\left(s_{j}, t_{j}\right)} \quad \text { (by (2)) } \\
& \left.\geqq \frac{\left|a_{j}\right| r_{j}^{\beta_{j}}}{\left(2 r_{j}\right)^{\alpha}} \quad \text { (by definition of } r_{j} \text { and } B_{j}\right) \\
& =\frac{\left|a_{j}\right|}{2^{\alpha}} r_{j}^{\beta_{j}-\alpha} \geqq \frac{\left|a_{j}\right|}{2^{1+\alpha}} .
\end{aligned}
$$


$j$ was arbitrary, so $\left\|f_{a}\right\|_{d^{\alpha}} \geqq\|a\| / 2^{1+\alpha}$. Hence, $a \rightarrow f_{a}$ is an isomorphism of $l_{\infty}$ onto its range.

Now, suppose $a \in c_{0},\|a\| \leqq 1$, and let $\varepsilon>0$ be given. There is $N$ so that if $i \geqq N,\left|a_{i}\right|<\varepsilon$. Pick $\lambda>0$ such that $\lambda<\delta / 2$ and $\lambda^{\beta_{i}-\alpha}<\varepsilon$ for $1 \leqq i<N$. Let $0<d(s, t)<\lambda$. It then follows that

$$
\frac{\left|f_{a}(s)-f_{a}(t)\right|}{d^{\alpha}(s, t)}<\varepsilon
$$

Hence, $f_{a} \in \operatorname{lip}\left(S, d^{\alpha}\right)$ when $a \in c_{0}$.

If $f \in \operatorname{Lip}\left(S, d^{\alpha}\right)$, define

$$
P f=\sum a_{n} f_{n} \quad \text { where } a_{n}=\frac{f\left(s_{n}\right)-f\left(t_{n}\right)}{f_{n}\left(s_{n}\right)} .
$$

If $\|f\| \leqq 1$, then

$$
\begin{aligned}
\left|f\left(s_{n}\right)-f\left(t_{n}\right)\right| & \leqq d^{\alpha}\left(s_{n}, t_{n}\right)=\left(2 r_{n}\right)^{\alpha} \\
& \leqq 2^{\alpha} d^{\alpha}\left(s_{n}, \widetilde{B}_{n}\right) \leqq 2^{1+\alpha} d^{\beta_{n}}\left(s_{n}, \widetilde{B}_{n}\right) \\
& =2^{1+\alpha} f_{n}\left(s_{n}\right),
\end{aligned}
$$

since $d^{\beta_{n}-\alpha}\left(s_{n}, \widetilde{B}_{n}\right) \geqq \frac{1}{2}$ by our choice of $\beta_{n}$. Hence, $\left|a_{n}\right| \leqq 2^{1+\alpha}$ for each $n$. Therefore, $P$ is a bounded linear mapping onto the image of $l_{\infty}$, and it is not hard to see that $P^{2}=P$.

Finally, let $f \in \operatorname{lip}\left(S, d^{\alpha}\right)$ and $a_{n}$ be as above. Also as above, we have

$$
d^{\alpha}\left(s_{n}, t_{n}\right) \leqq 2^{1+\alpha} d^{\beta_{n}}\left(s_{n}, \widetilde{B}_{n}\right)=2^{1+\alpha} f_{n}\left(s_{n}\right),
$$

so

$$
\left|a_{n}\right|=\frac{\left|f\left(s_{n}\right)-f\left(t_{n}\right)\right|}{f_{n}\left(s_{n}\right)} \leqq 2^{1+\alpha} \frac{\left|f\left(s_{n}\right)-f\left(t_{n}\right)\right|}{d^{\alpha}\left(s_{n}, t_{n}\right)} \rightarrow 0,
$$

Thus, $\left\{a_{n}\right\} \in c_{0}$. This completes the proof for $\alpha<1$.

To show that $l_{\infty}$ can be embedded in $\operatorname{Lip}(S, d)$, observe that nothing changes up to our choice of $\beta_{j}$. Now, our choice will satisfy the same requirements except $\beta_{j}>\alpha=1$ for each $j$. Note that since $\left|x^{p}-y^{p}\right|$ $\leqq p|x-y|$ for all $x, y \in[0,1], p \geqq 1$, we have $\left\|f^{p}\right\|_{d} \leqq p\|f\|_{d}$ if $f \in \operatorname{Lip}(S, d)$, $0 \leqq f \leqq 1$. Hence, we may verify that $\left\|f_{a}\right\|_{d} \leqq\|a\| \max (1,4 / \delta)$ while $\left\|f_{a}\right\|_{\infty} \leqq\|a\|$. Also, just as before, $\left\|f_{a}\right\|_{d} \geqq \frac{1}{4}\|a\|$. This completes the proof.

Before closing, let us mention that if there is a $\delta>0$ and a partition $\mathscr{P}$ of $S$ such that $d(A, B) \geqq \delta$ for $A, B \in \mathscr{P}, A \neq B$, then $\operatorname{lip}\left(S, d^{\alpha}\right), 0<\alpha \leqq 1$, contains a subspace isomorphic to $l_{\infty}(\mathscr{P})$. Given $\phi \in l_{\infty}(\mathscr{P})$, simply define $f_{\phi}(s)=\phi(A)$ when $s \in A \in \mathscr{P}$. Then $\phi \rightarrow f_{\phi}$ is a bicontinuous linear map- 
ping of $l_{\infty}(\mathscr{P})$ into $\operatorname{lip}\left(S, d^{\alpha}\right)$. For example, if

$$
S=\{(n, 0): n=1,2, \ldots\} \cup\{(n, 1 / n): n=1,2, \ldots\}
$$

in the plane, then $\operatorname{lip}\left(S, d^{\alpha}\right)$ contains a complemented subspace isomorphic to $l_{\infty} \oplus c_{0}$. It, thus, seems natural to conjecture that $\operatorname{lip}\left(S, d^{\alpha}\right)$ is, in general, an arbitrary direct sum of subspaces isomorphic to $c_{0}(\Gamma)$ and $l_{\infty}(\Gamma)$. In particular, it is still an open question whether $\operatorname{lip}\left(S, d^{\alpha}\right)$ is isomorphic to $c_{0}$ when $S$ is compact and $0<\alpha<1$.

\section{REFERENCES}

1. R. Bonic, J. Frampton and A. Tromba, $\Lambda$-manifolds, J. Functional Analysis 3 (1969), 310-320. MR 38 \#6635.

2. M. M. Day, Normed linear spaces, 2nd rev. ed., Academic Press, New York; SpringerVerlag, Berlin, 1962. MR 26 \# 2847.

3. J. A. Johnson, Banach spaces of Lipschitz functions and vector-valued Lipschitz functions, Trans. Amer. Math. Soc. 148 (1970), 147-169.

DePartment of Mathematics and Statistics, OKlahoma State University, StillWATER, OKLAHOMA 74074 